\title{
A Novel Sensor to Measure the DC Bias at the Point of Common Coupling of Distribution Transformers
}

\author{
H.M.A.I. Herath and J.P. Karunadasa
}

\begin{abstract}
With the proliferation of solar PV inverters and other power electronic intensive loads on the power utility system the accumulated DC current at the point of common coupling can be significant. This DC current disturbs the normal operation of the distribution transformer due to the change of symmetrical magnetization, leading to significant power quality issues upstream in addition to the effects on the transformer itself. Therefore, measures need to be adopted to divert DC current away from the secondary of the transformer. Accurate sensing of DC current at the transformer secondary is a prime need in any implementation of a DC current diversion scheme. This is a challenging task because the DC current is superimposed with a large AC current. In this paper, a novel design of a current sensor that can accurately detect small DC current superimposed on a large AC current at the $400 \mathrm{~V}$ secondary of a distribution transformer is presented. The sensor is fast responsive and suitable for closed loop control of DC current. The details of sensor design and modelling are presented. The sensor performance is simulated in MATLAB and the simulation results are also given.
\end{abstract}

Keywords: Distribution Transformer, DC current sensing, Magnetizing current, Power quality, PCC

\section{Introduction}

Grid-tie inverters, power electronic based loads and other non-linear loads on the utility system create small amounts of DC current on top of their normal AC current. Due to heavy proliferation of such inverters and loads on the present day utility system, the net DC current accumulated at the secondary of the distribution transformer is significant in relation to its magnetizing current. This DCcurrent offsets the operating point of the magnetization characteristic for the iron core, and creates a severe asymmetry between the positive and negative half cycles of the magnetization current, both in magnitude and shape, owing to magnetic saturation. It directly affects the shape of input current, and also reproduces a DC current at the input with magnitude even higher than that present at the secondary[1]. The transformer itself is also affected by the action of DC current[2], [3].

Elimination of DC current at the secondary of the distribution transformer is addressed in several stages.

(i) Restricting DC current produced by equipment by adopting appropriate guidelines during their designs. (ii) Using interconnections that can stop DC current flowing in to the power network.

(iii) Deploying DC current diversion systems at the transformer secondary.

Influential equipment, such as grid-tie inverters adopt International or country-wide regulations to limit the maximum DC current. Table 1 presents the existing status of guidelines and regulations enforced in five selected countries in respect of grid-tie inverters.

Line frequency transformer interconnection, high frequency transformer interconnection and transformerless interconnection are examples of interconnections that stop DC current flowing in to the power network [2] [7], [9].

DC current diversion from the transformer secondary is the sure option to address all residual problems associated with the DC current. The implementation of such a current diversion system is inherently based on power electronics with closed loop current control.

Eng. (Ms.) H.M.A.I. Herath, C. Eng., MIE(SL), B.Sc. Eng. (Hons) (Ruhuna), M.Sc. (Moratuwa), Lecturer, General Sir John Kotelawala Defence University, Rathmalana, Sri Lanka. Email:ayeshaih@gmail.com.

Eng. (Prof.) J.P. Karunadasa, C. Eng., MIE(SL), B.Sc. Eng. (Hons) (Moratuwa), M.Sc. (Manchester), Ph.D. (Manchester). Associate Professor in Electrical Engineering, University of Moratuwa.

Email:karu@elect.mrt.ac.lk. 
Table 1 - Limits of DC current injection permitted by different countries for grid-tie inverters (LV system)

\begin{tabular}{|l|l|l|}
\hline Country & Standard & $\begin{array}{l}\text { Maximum permitted } \\
\text { DC current }\end{array}$ \\
\hline $\begin{array}{l}\text { United } \\
\text { Kingdom }\end{array}$ & ER G83/1 & $\begin{array}{l}0.25 \% \text { of rated } \\
\text { current of the inverter }\end{array}$ \\
\hline Australia & AS 4777.2 & $\begin{array}{l}0.5 \% \text { of rated current } \\
\text { of the inverter or } 5 \mathrm{~mA} \\
\text { whichever greater }\end{array}$ \\
\hline USA & $\begin{array}{l}\text { IEEE 929- } \\
\text { IEEE 1547 }\end{array}$ & $\begin{array}{l}\text { 0.5\% of rated current } \\
\text { of the inverter } \\
0.5 \% \text { of rated current } \\
\text { of the inverter }\end{array}$ \\
\hline Switzerland & IEC 61727 & $\begin{array}{l}\text { 1\% of rated current of } \\
\text { the inverter }\end{array}$ \\
\hline Germany & $\begin{array}{l}\text { DINVDE } \\
0126-1-1\end{array}$ & $\begin{array}{l}\text { 1A (Total injection) } \\
\text { [States in the case of } \\
\text { DC current injection } \\
\text { greater than 1A, } \\
\text { disconnection is } \\
\text { mandatory in 0.2S] }\end{array}$ \\
\hline
\end{tabular}

A sensor that can accurately measure small DC current superimposed with a large AC current is a need for this task. Use of a Hall-element based current sensor is not suitable due to the noise and inherent drifts of the sensor itself which makes the extraction of the weak DC current information very difficult and inaccurate. A better option would be to sense the DC voltage across the transformer secondary produced by the DC current in the secondary-resistance. One arrangement proposes to take the full voltage across the secondary and to remove the AC part with an equal and opposite $\mathrm{AC}$ component induced with an instrument transformer [3]. However, a proper cancellation of the AC component is not possible with a real instrument transformer and moreover the instrument transformer itself is subject to DC effects leading to significant measuring errors. Another proposal suggested is to use a small instrument transformer across the secondary and to use its magnetizing current to extract the information of DC voltage by processing the asymmetry between its positive and negative half-cycles [7], [8]. This method is relatively complex to implement and the accuracy depends largely on the methods used to acquire and process the magnetizing current signal.

The DC current sensor proposed in this paper does not use an instrument transformer. It is a voltage based sensor with a special AC cancellation technique. The front end is a 2stage RC divider with intermediate RLC filter stage, and a final gain setting stage. The sensor is found to be performing exceptionally well on a $400 \mathrm{~V}$ AC secondary with a superimposed DC voltage component of $1.25 \mathrm{~V}$ DC voltage.

\section{DC Current in a Sample Distribution System}

In order to show that a distribution transformer in the present day power distribution system is experiencing a significant DC current component at its secondary, a test measurement was made at the secondary of an $11 \mathrm{kV} / 400 \mathrm{~V}$, $400 \mathrm{kVA}, \quad 50 \mathrm{~Hz}$ three-phase distribution transformer feeding part of the University. Measurements were carried out using FLUKE 435 Power Quality Analyser and Figure 1 gives a screen-shot of basic AC values. Figure 2 gives the spectrum of currents which indicates DC currents of $1.1 \mathrm{~A}, 1.5 \mathrm{~A}$ and $1.4 \mathrm{~A}$ in three phases, which are of the order of $10 \%$ of the magnetizing current for the transformer.

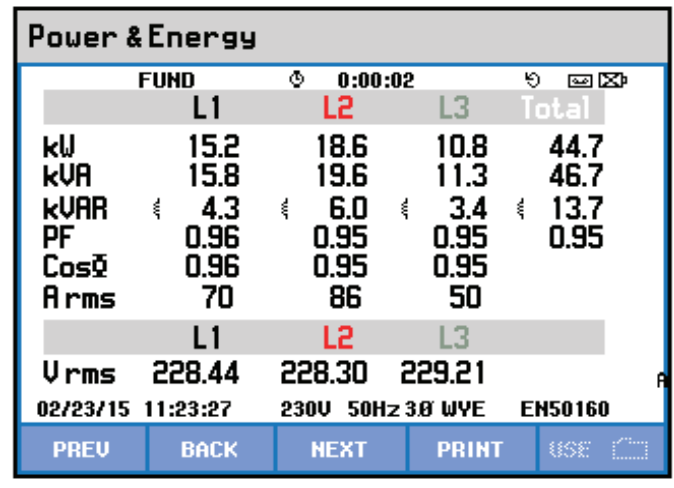

Figure 1 - Screen shot of Power Analyser on $A C$ values at the transformer secondary

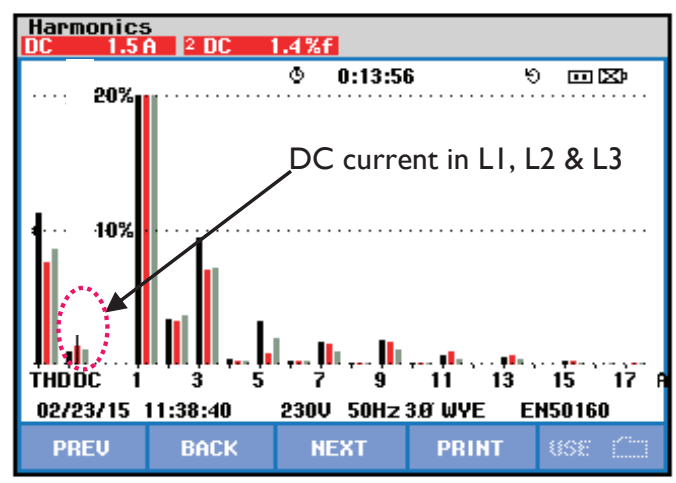

Figure 2 - Current spectrum at the transformer secondary 


\section{DC Current Sensor}

DC current flowing in the transformer secondary creates a corresponding DC voltage component across the secondary-resistance, so by measuring the secondary voltage, it is possible to extract this DC component easily. This is considered a better option than sensing DC current directly because Voltage can be sensed more accurately than current, especially very small values of current.

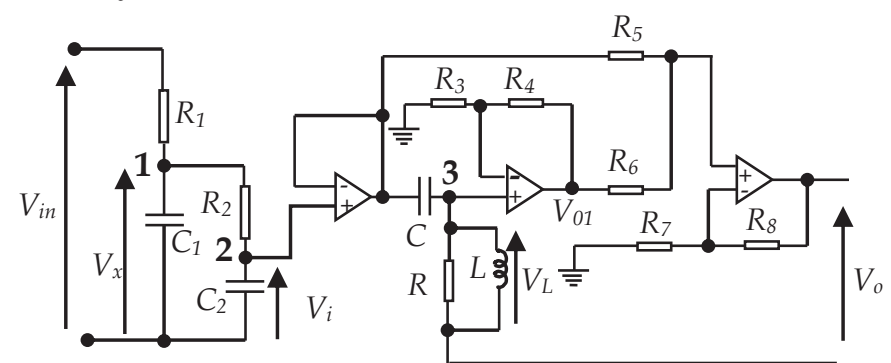

Figure 3 - DC current detector circuit

Figure 3 shows the arrangement of the proposed DC current sensor. At the front end, it uses a two-stage $R C$-divider to attenuate the AC voltage component in the measuringvoltage without affecting the magnitude of the superimposed DC component. Values of $R$ and $C$ components in the voltage divider were designed to lower the AC component from 400 $\mathrm{V}$ down to about $2 \mathrm{~V}$ with a response time near 0.5 second. Body resistances of the capacitors tend to create a small attenuation of the DC component but this is predictable and can be accommodated in the design. The residual AC voltage component present in the output of the $\mathrm{RC}$ divider is then removed by the $\mathrm{AC}$ cancellation circuit which uses an RLC low-pass filter at the center. The separated DC component is finally taken through the gainsetting stage to restore the path attenuations.

Signal output from the RC divider stage was buffered before passing it on to the AC cancellation circuit. This was done to avoid the signal getting distorted due to the loading effects and also for the safety of the processing hardware.

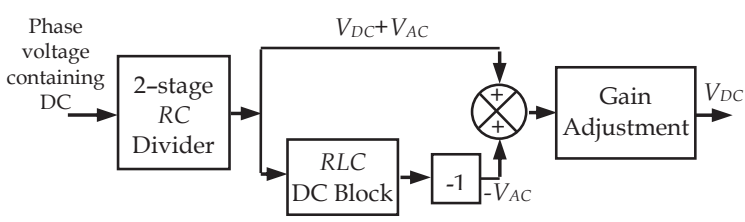

Figure 4 - Functionality of DC current detector
Figure 4 illustrates the functionality of the current sensor. The AC cancellation circuit takes the input signal along two parallel paths. One path contains an RLC filter which drops the DC component in the signal and offers a gain of -1 to the remaining signal. The other path allows the signal to flow without a change. The signals so arriving on two paths are added together so that the output becomes the DC component only. Positive gain stage at the end restores the magnitude of the output to match it with the actual DC voltage present at the input against slight attenuations, if any.

\subsection{Transfer Function of DC Current Sensor}

Transfer function of the $R C$-divider stage with ideal capacitances is obtained as,

$\frac{V_{i}}{V_{\text {in }}}=\frac{1}{S^{2} C_{1} C_{2} R_{1} R_{2}+S\left(C_{1} R_{1}+C_{2} R_{2}+C_{2} R_{1}\right)+1}$

The modified transfer function $\mathrm{G}_{1}(\mathrm{~s})$, assuming a leakage resistance $r$ for each capacitance is found as,

$$
\begin{aligned}
& G_{1}(s)=\frac{V_{i}}{V_{i n}} \\
& =\frac{1}{S^{2} C_{1} C_{2} R_{1} R_{2}+S\left(C_{1} R_{1}+C_{2} R_{2}+C_{2} R_{1}+\frac{R_{1} R_{2}}{r}\left(C_{1}+C_{2}\right)\right)} \\
& \quad+\left(1+\frac{2 R_{1}}{r}+\frac{R_{2}}{r}+\frac{R_{1} R_{2}}{r^{2}}\right)
\end{aligned}
$$

Similarly, transfer function $G_{2}(s)$ of the RLC filter path of the AC cancellation circuit is obtained as,

$G_{2}(s)=\frac{V_{o 1}}{V_{i}}=\frac{\left(1+\frac{R_{4}}{R_{3}}\right) S^{2}}{S^{2}+\frac{S}{R C}+\frac{1}{L C}}$

Overall transfer function $\mathrm{G}(\mathrm{s})$ of the current detector is thus obtained as,

$G(s)=\frac{V_{o}}{V_{\text {in }}}=\left(1+\frac{R_{8}}{R_{7}}\right)\left(\frac{1+G_{2}(s)}{2}\right) G_{1}(s)$

Specific transfer functions for the actual values of the components (shown later in Figure 7) are thus found as follows:

$$
\begin{aligned}
& G_{2}(s)=\frac{1689 S^{2}}{S^{2}+166.7 S+166666666.7} \\
& G_{1}(s)=\frac{1000}{3 S^{2}+173.8 S+1108.1} \\
& G(s) \\
& =\frac{1115\left(1690 S^{2}+166.7 S+166666666.7\right)}{\left(S^{2}+166.7 S+166666666.7\right)\left(3 S^{2}+173.8 S+1108.1\right)}(7)
\end{aligned}
$$


Gain of the path of RLC branch of the AC cancellation circuit is given by the value of G2(S). Its value at $S=0$ gives the DC gain and at $S=j 2 \pi \times 50 \mathrm{rad} / \mathrm{s}$ gives the $50 \mathrm{~Hz}$ gain. By substituting values they are found as,

$G_{2}(0)=0$

$G_{2}(j 2 \pi \times 50)=(-1.00+j 0.00)$

Thus, the specifications of zero gain for the DC and -1 gain for the $50 \mathrm{~Hz}$ signal are satisfied. Thus, in the steady state conditions the effects of $50 \mathrm{~Hz}$ AC voltage at the transformer secondary does not influence the sensor output.

During normal operation of the transformer, the secondary AC voltage is in a steady state and the DC voltage component may exhibit slow variations depending on the way the loads inject DC component of current in to the network. These changes are imitated at the sensor output with a brief and fast transient.

Figure 5 shows the response of transfer function $G(s)$ for a step test input of 1.25 V DC without the $\mathrm{AC}$ component, which reaches the steady state of $1.25 \mathrm{~V}$ in about 0.3 second. Figure 6 shows the response for a step input of $1.25 \mathrm{~V}$ DC with400 V AC (rms), which also reaches the steady state of $1.25 \mathrm{~V}$ after a brief transient. In the latter case the transient peak is several times greater than the steady state but this is not considered an issue as this case of step change of $400 \mathrm{~V}$ occurs only when the circuit is switched on. By using a signal limiter at the sensor output this one time transient peak of the response can be clipped out not to create unwanted influences on the succeeding stages that use the sensor output.

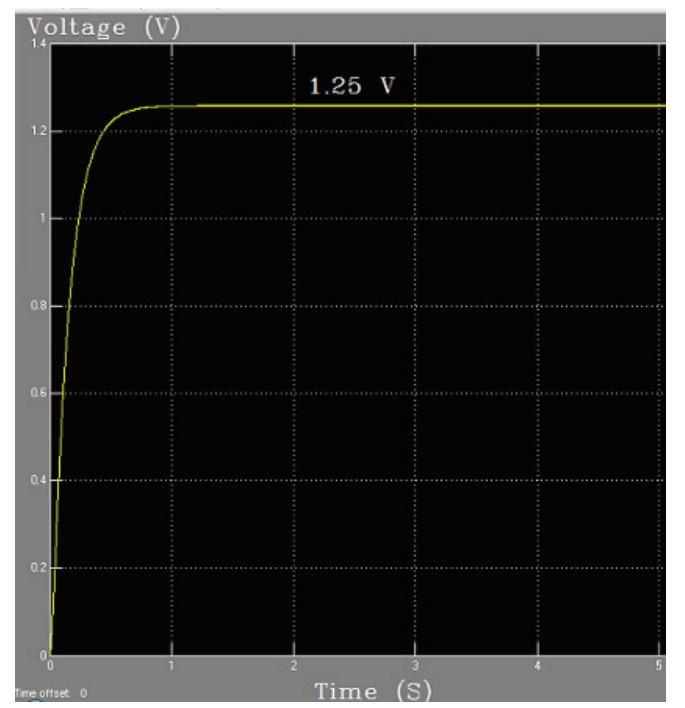

Figure 5 - Response of transfer function G(s) for a step input of $1.25 \mathrm{~V}$ DC without $\mathrm{AC}$

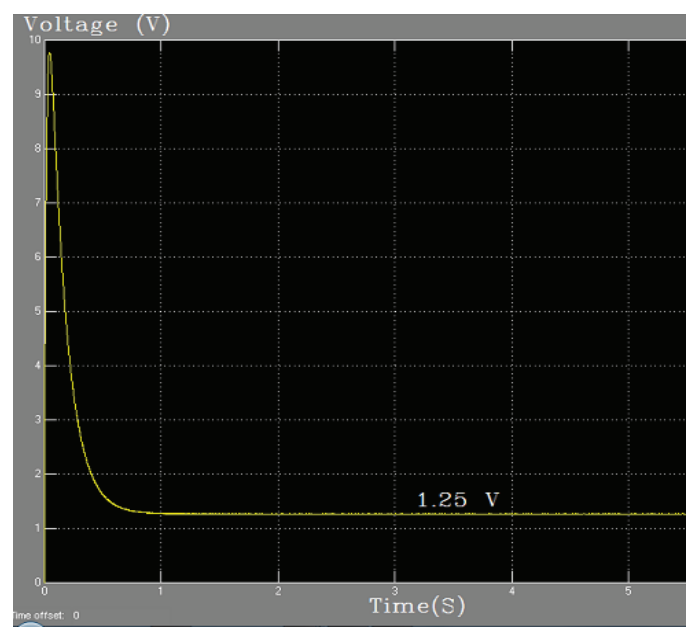

Figure 6 - Response of transfer function G(s) for a step input of $1.25 \mathrm{~V}$ DC with $400 \mathrm{~V} \mathrm{AC}$

\subsection{Response of the DC Current Sensor}

DC current sensor circuit was simulated in MATLAB and a series of tests were conducted to assess the sensor performance. Practical capacitors $C_{1}$ and $C_{2}(30 \mu \mathrm{F}$ each) used in the $R C$ divider stage have some finite leakage resistances and to account for this effect a leakage resistance of $53 \mathrm{k} \Omega$ (which is typical for a $30 \mu \mathrm{F}$ capacitor) was used across each capacitor in the simulation. This enabled a better adjustment of the gain of the final stage of the sensor to trim out stray effects and obtain correct magnitude of the sensor output equal to the actual DC voltage superimposed in the transformer secondary.

Figure 7 shows the simulation model for the sensor. Figure 8 shows the sensor output corresponding to a step test input of $1.25 \mathrm{~V}$ (DC) without the AC voltage component. Clearly, it matches the corresponding response in Figure 5 found from the transfer function analysis. Similarly, Figure 9 shows the sensor output corresponding to step test input of 1.25 $\mathrm{V}$ (DC) with $400 \mathrm{~V}$ (AC rms) at the input, which again matches the corresponding response in Figure 6 found from the transfer function analysis.

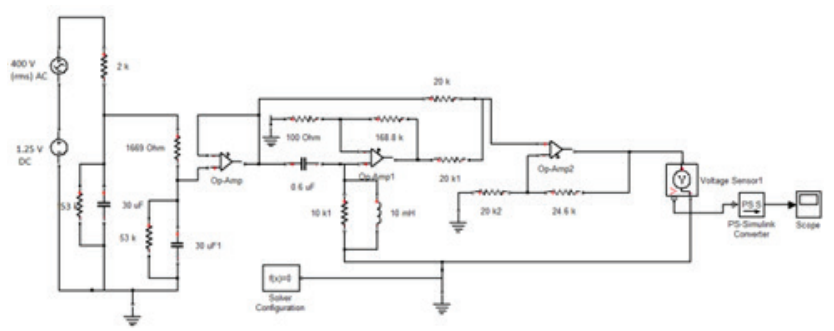

Figure 7 - MATLAB simulation model with $1.25 \mathrm{~V}(\mathrm{DC})$ and $400 \mathrm{~V}(\mathrm{AC})$ at sensor input 


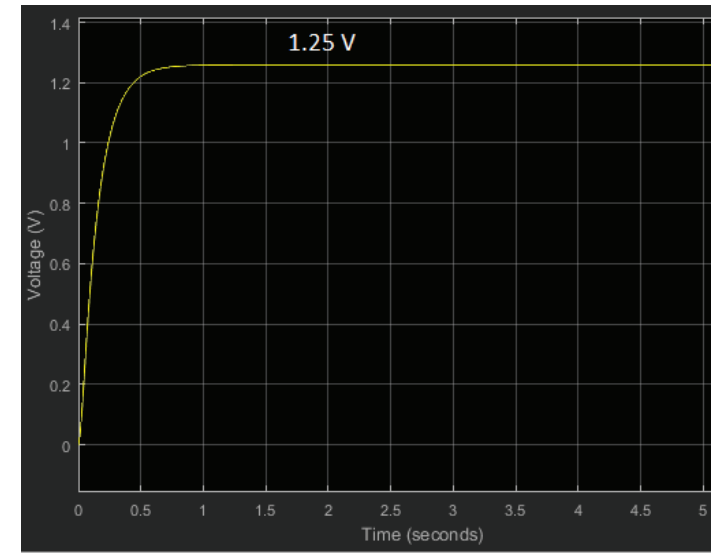

Figure 8 - Sensor output for 1.25 V DC step input without $A C$ voltage

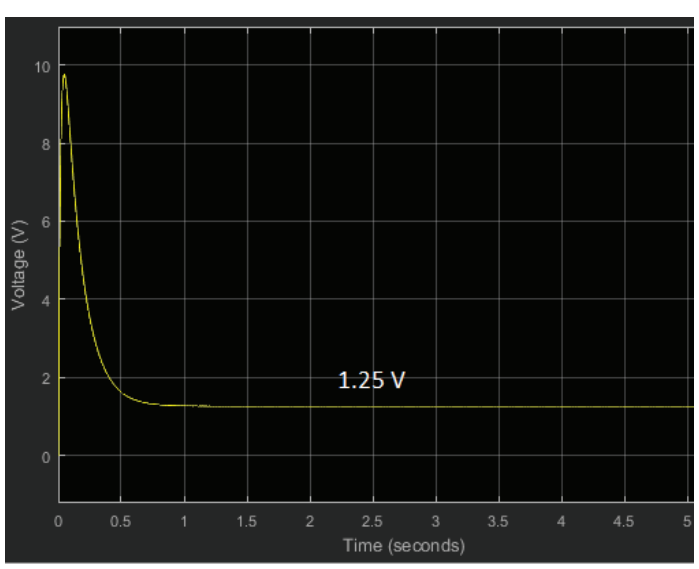

Figure 9 - Response of the current Sensor for step input of $1.25 \mathrm{~V}$ DC superimposed with $400 \mathrm{~V}(\mathrm{AC})$

In Figure 9, the initial inrush peak observed in the sensor output is due to the sudden appearance of the $400 \mathrm{~V} \mathrm{AC}$ at the sensor input. This is an unwanted and one time response which could be dropped by means of a signal limiter at the sensor output. Figure 10 shows the sensor output for a step test input of $1.25 \mathrm{~V}$ (DC) which is brought back to zero stepwise at time $t=3 \mathrm{~s}$ with $400 \mathrm{~V}$ ACrms) at the input. It clearly shows that the $1.25 \mathrm{~V}$ to $0 \mathrm{~V}$ negative step at 3 second does not create an inrush. This is because, by the time of 3 seconds the initial inrush due to the $\mathrm{AC}$ input has died out and any subsequent changes in the DC component produces only its own response. This means, after the initial switch-on, the sensor is completely blind to the $\mathrm{AC}$ voltage present at the sensor input and the DC voltage present at the sensor input solely produces the response. Even a minute change in the DC voltage present at the sensor input is detected with fast speed of response. This is unique feature of this new sensor.

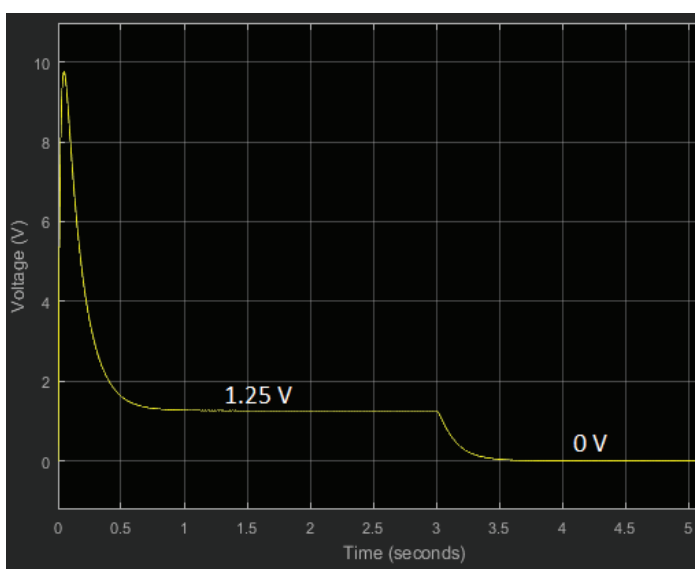

Figure 10 - Response of the current Sensor for step input of 1.25 V DC, followed by step down to zero at $t=3 \mathrm{~s}$, with $400 \mathrm{~V}$ (AC)

During normal operation of a transformer the AC voltage waveform can undergo sags or swells time to time. In order to investigate the action of the sensor on voltage sags and swells, the sensor was simulated for $\pm 10 \%$ variation of the rms value. Figure 11 shows the sensor response for $10 \%$ step rise of $\mathrm{AC}$ voltage at $\mathrm{t}=3$ second, after an initial switching onto nominal $400 \mathrm{~V}$ at $\mathrm{t}=0$ without a DC component. It indicates that the sensor is producing just a momentary response on the swell. Similarly, Figure 12 shows the sensor response for a step voltage sag of $10 \%$ at $t=3$ after initial switching onto $400 \mathrm{~V} \mathrm{AC}$ at $\mathrm{t}=0$ without a DC. It too shows that the sensor is producing just a momentary response.

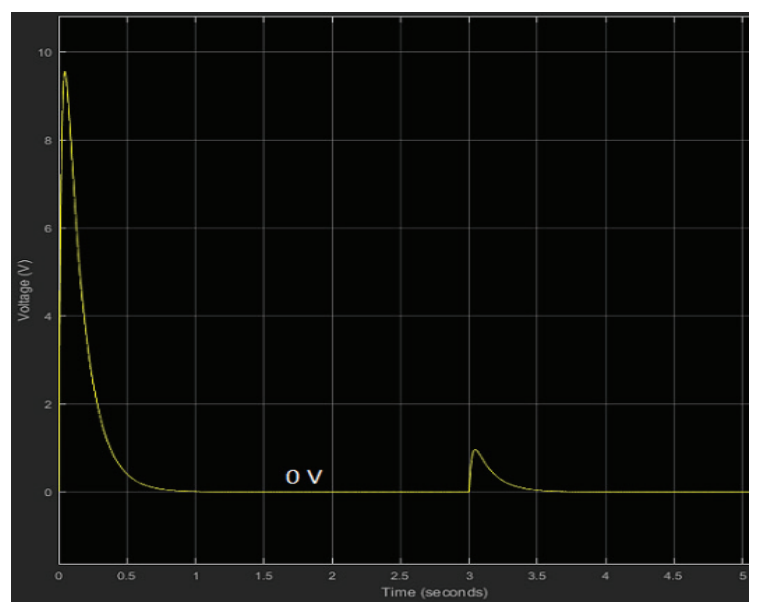

Figure 11 - Response of the current Sensor for $10 \%$ step swell of AC voltage at $t=3 \mathrm{~s}$, after initial switching onto $400 \mathrm{~V}$ (AC) 


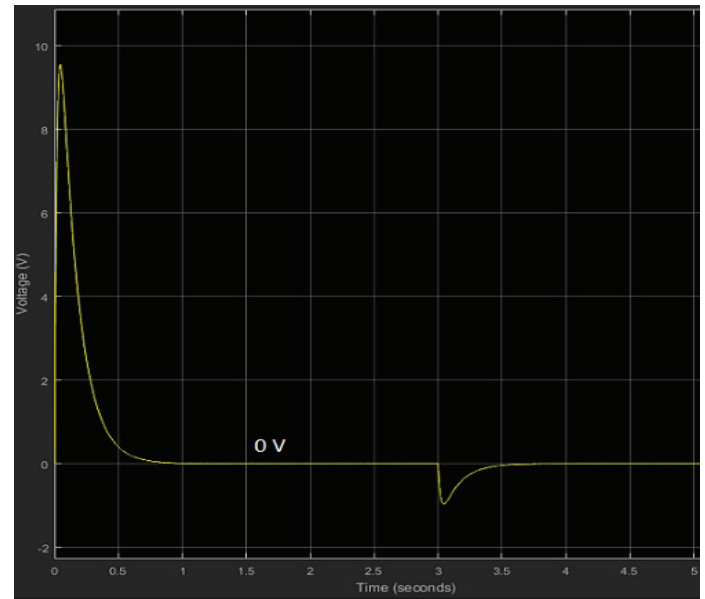

Figure 12 - Response of the current Sensor for $10 \%$ step sag of AC voltage at $t=3 \mathrm{~s}$, after initial switching onto $400 \mathrm{~V}$ (AC)

\section{Conclusion}

Development of a reliable sensor to detect small DC current values present at the secondary side of a distribution transformer has been a challenge. This was mainly due to the large AC current and the large $\mathrm{AC}$ voltage present at the secondary. Magnetic cores based detections proved inaccurate due to the DC magnetization of the cores themselves unless special techniques are used to model such behaviours.

This paper presented a new sensor without involving magnetic cores to detect very small DC current values in a $400 \mathrm{~V}$ transformer secondary. Measuring input has been the DC voltage developed between the secondary terminals due to the DC current in the secondary-resistance. This small DC voltage is riding on $400 \mathrm{~V}$ AC component but the characteristic differences between AC and DC could inflict different levels of attenuations to weaken the AC component with respect to the DC component. The sensor as a whole functions by eliminating the AC based signal component altogether with a special signal mixing stage. Performance of the sensor was theoretically established and validated through MATLAB simulations. The sensor was responding extremely well with a response time below 0.3 second for a step change of the DC current, and this timing is quite acceptable for any DC current elimination systems to be operative on the transformer. At the point of initial switching $\mathrm{ON}$ of the transformer, there is an inrush peak of the sensor output due to the $400 \mathrm{~V}$ AC inrush but with a signal limiter it could be clipped out without anyharm. The complete details of the sensor including component values, transfer functions, simulation model, and simulation results have been presented and these are useful for the reconstruction of the sensor, if necessary.

\section{Acknowledgement}

Author wishes to acknowledge the Head of the Department of Electrical Engineering at the University of Moratuwa for providing resources to conduct the research.

\section{References}

1. Karunadasa, J. P., Herath, H. M. A. I., "Investigation of the Effects of DC Current at the Point of Common Coupling on the Operation of Distribution Transformers", Proceedings of the 4th International Research conference, General Sir John Kotelawala Defence University, Sri Lanka, August 2016.

2. Atkinson, D. J., Blewitt, W. M., Kelly, J., Lakin, R. A., Approach to Low Cost Prevention of DC Injection in Transformerless Grid Connected inverters, IET Power Electronics, 2010, Vol. 3, Iss.1, pp. 111-119.

3. Ahfock, A., and Bowtell, L., Direct Current Offset Controller for Transformerless Single Phase Photovoltaic Grid-Connected Inverters, IET Renewable Power Generation, 2010 Vol. 4, Iss.5, pp.428-437.

4. Haeberlin, H., Evolution of Inverters for Grid Connected Systems from 1989 to 2000, 17th European Photovoltaic Solar Energy Conference, 2001.

5. Armstrong, M., Atkinson, D. J, Johnson, C. M., \& Abeyasekera, T. D., “Auto Calibrating DC Link Current Technique for Transformerless Grid Connected H-bridge Inverter Systems", IEEE Transactions on Power Delivery, Vol. 21, No. 5, September 2006.

6. Alonso, M., Barrado, A., and Chenlo, F., et al(2006) DC Current Injection into the Network from PV Grid Inverters, IEEE Transaction on Power Delivery.

7. GiampaoloButicchi, Emilio Lorenzani, Giovanni Franceschini“ A DC Offset Current Compensation Strategy in TransformerlessgrodConnected Power Converters", IEEE Transactions on Power Delivery, Vol. 26, No. 4, October 2011. 
8. GiampaoloButicchi, \& Emilio Lorenzani, "Detection Method of the DC Bias in Distribution Power Transformers", IEEE Transactions on Industrial Electronics, Vol. 60, No. 8, August 2013.

9. Fronius International, "High frequency transformer with transformer switchover", https://www.fronius.com/cps/rde/xbcr/SID-

$4725 B B 5 C-$

2C0F62DA/fronius_international/SE_TA_High_Freq uency_Transformer_With_Transformer_Switchover_ EN_320487_snapshot.pdf, Visited, 20 th February 2015. 